\title{
Encefalopatía posterior reversible en niño trasplantado de corazón
}

\author{
Posterior reversible encephalopathy in a child transplanted of
}

heart

\author{
Isabel Cristina Zuluaga-Aristizábal', Marcela Zuluaga-Restrepo², Margarita Zapata- \\ Sánchez ${ }^{3}$, Arnaldo Andrés Palomino-Rodríguez ${ }^{4}$, Diana Patricia Restrepo-Bernal5 ${ }^{5}$ LLAC
}

Fecha correspondencia:

Recibido: octubre 21 de 2020. Revisado: noviembre 5 de 2020. Aceptado: enero 22 de 2021.

Forma de citar:

Zuluaga-Aristizábal IC, ZuluagaRestrepo M, Zapata-Sánchez M, Palomino-Rodríguez AA, RestrepoBernal DP. Encefalopatía posterior reversible en niño trasplantado de corazón. Rev CES Med. 2021; 35(1): 37-43.

\section{Open access}

(C) Derecho de autor Licencia creative commons

Ética de publicaciones

Revisión por pares

Gestión por Open Journal System DOl: http://dx.doi.org/10.21615/ cesmedicina.35.1.4

ISSN 0120-8705

e-ISSN 2215-9177

\section{Resumen}

La encefalopatía posterior reversible es un síndrome clínico-radiológico caracterizado por hipertensión arterial, cefalea, alteraciones visuales, convulsiones y delirium. Radiológicamente, se observa edema vasogénico en regiones subcorticales con predilección por los lóbulos posteriores del cerebro. Ha sido asociada con trasplante de órgano sólido, hipertensión arterial y uso de medicamentos inmunosupresores, como los anticalcineurínicos. Se reporta el caso de un niño de seis años quien inició con cefalea, cifras tensionales elevadas, irritabilidad, agresividad y por momentos agitado cinco días después de recibir un trasplante ortotópico de corazón y un día después de inicio de tacrolimus. El tratamiento incluyó manejo sintomático con antihipertensivos y anticonvulsivantes, además del aumento progresivo del inmunosupresor hasta niveles terapéuticos en sangre para disminuir el riesgo de rechazo del injerto. El síndrome de encefalopatía posterior reversible debe ser considerado en pacientes trasplantados de corazón que reciben inhibidores de la calcineurina y presentan síntomas neurológicos.

Palabras clave: Síndrome de leucoencefalopatía posterior; Trasplante; Corazón; Preescolar; Tacrolimus.

\footnotetext{
Abstract

Posterior reversible encephalopathy is a clinic-radiological syndrome characterized by arterial hypertension, headache, visual disturbances, seizures and delirium. Radiologically, vasogenic edema is observed in subcortical regions with predilection for the posterior lobes of the brain. It has been associated with solid organ transplantation, arterial hypertension and the use of immunosuppressive drugs such as anticalcineurin. We report the case of a six-year-old boy who started with headache, elevated blood pressure, irritability, aggressiveness and at times agitated five days after receiving an orthotopic heart transplant and one day after starting tacrolimus. His treatment included symptomatic management with antihypertensives and anticonvulsants, in addition to a progressive increase in immunosuppressant to therapeutic blood levels to reduce the risk of graft rejection. Reversible posterior encephalopathy syndrome should be considered in heart transplant recipients receiving calcineurin inhibitors who present neurological symptoms.
} 
Enero - abril de 2021 - Pág 38

\section{Sobre los autores:}

1. Estudiante de Pregrado de Medicina, Universidad CES, Medellín-Colombia.

2. Residente de III año de Psiquiatría, Universidad CES, Medellín-Colombia.

3. Pediatra Cardióloga, Coordinadora del Programa de Trasplante Pediátrico, Clínica CardioVID, Coordinadora del Postgrado de Cardiología Pediátrica Universidad Pontificia Bolivariana, MedellínColombia.

\section{Fellow de Cardiología Pediátrica, Universidad Pontificia Bolivariana, Clínica CardioVID, Medellín- Colombia.}

5. Psiquiatra de Enlace, MSc. Epidemiología. Docente de pre y post grado de psiquiatría Universidad CES. Medellín, Colombia.

En niños trasplantados de corazón se ha reportado una prevalencia del síndrome de encefalopatía posterior reversible de 5,5\% y mayor predominio en mayores de cinco años, quienes serían más susceptibles por el estrecho margen que toleran de la presión arterial. Como factores de riesgo se identifica el efecto tóxico de los anticalcineurínicos, que pueden dañar el endotelio vascular cerebral y producir hipertensión arterial.
Keywords: Posterior Leukoencephalopathy Syndrome; Heart Transplantation; Child Preschool; Tacrolimus.

\section{Introducción}

El síndrome de encefalopatía posterior reversible es una entidad clínico-radiológica que se presenta en niños y adultos. Fue descrita por Hinchey et al. en 1996 a partir de una serie de pacientes adultos que presentaron convulsiones, cefalea, compromiso del nivel de conciencia, alteraciones visuales y déficit motor, y en quienes se identificó edema vasogénico en regiones posteriores del cerebro, con predominio de regiones parieto-occipitales, aunque también se ha descrito compromiso de los lóbulos frontal, temporal y menos frecuente en el tallo cerebral, tálamo, ganglios basales y cerebelo (1-3).

Las condiciones clínicas más frecuentemente asociadas son hipertensión arterial, diferentes enfermedades (renal, autoinmune, neoplásica y hematológica), así como el uso de medicamentos como los inmunosupresores (ciclosporina y tacrolimus) (4). El mayor compromiso de las regiones cerebrales posteriores se ha atribuido a la menor capacidad de autorregulación de la vasculatura de estas zonas, donde hay menos inervación simpática (5).

Los hallazgos radiológicos, además de los clínicos, son necesarios para realizar el diagnóstico. La tomografía cerebral, útil en algunos casos, es superada por la resonancia magnética $(4,6)$. La mayoría de los casos son reversibles, por lo que la sospecha clínica y el diagnóstico temprano pueden evitar secuelas permanentes como hemorragia, infarto cerebral y la muerte $(1,6)$.

En niños trasplantados de corazón se ha reportado una prevalencia de 5,5 \% y mayor predominio en mayores de cinco años (1), quienes serían más susceptibles por el estrecho margen que toleran de elevación en la presión arterial (4). Dentro de los factores de riesgo se identifica el efecto tóxico de los anticalcineurínicos, como ciclosporina y tacrolimus, que pueden dañar el endotelio vascular cerebral y producir hipertensión arterial $(1,7)$.

El objetivo de este reporte es presentar el caso clínico de un niño de seis años, trasplantado de corazón, el cual a los cinco días postrasplante presentó un cuadro clínico y radiológico compatible con síndrome de encefalopatía posterior reversible. Este manuscrito, se preparó siguiendo las recomendaciones del grupo CARE para reporte de casos (8).

\section{Presentación del caso}

Un niño de seis años recibió trasplante ortotópico de corazón por antecedente de miocarditis viral ocurrida a los tres meses de vida, con posterior deterioro de la función ventricular y falla cardíaca, que progresó en los siguientes cinco años hasta requerir hospitalizaciones frecuentes, dependencia de inotrópicos y necesidad de cardio-desfibrilador por alto riesgo de muerte súbita. El trasplante fue realizado con éxito y el paciente fue extubado a las 24 horas postrasplante en cuidados intensivos.

El primer día postoperatorio presentó hipertensión arterial sistémica, la cual fue controlada con verapamilo; además, presentó disminución de la atención y tendencia a la agitación psicomotora, por lo cual se hizo diagnóstico de delirium hiperactivo, que fue manejado con quetiapina. La inmunosupresión fue iniciada con timoglobulina, micofenolato y prednisolona. 
Una semana después de haber iniciado el cuadro clínico, una resonancia magnética cerebral mostró edema citotóxico parieto-occipital izquierdo, la cual confirmó el diagnóstico de síndrome de encefalopatía posterior reversible.
Al cuarto día posoperatorio se inició tacrolimus $0,5 \mathrm{mg}$ cada 12 horas y al día siguiente comenzó con cefalea, cifras tensionales elevadas manejadas con nitroprusiato de sodio de forma aguda y posteriormente verapamilo y enalapril, como mantenimiento. Se determinó que estaba cursando un estado epiléptico no convulsivo, dado por alteración del nivel de conciencia, apertura ocular espontánea sin contacto con el medio, nistagmus horizontal, sin respuesta verbal y relajación de esfínteres, por lo que se indicó midazolam intravenoso dosis única y bolo de fenobarbital como manejo abortivo, y una vez controlado el episodio continuó con levetiracetam intravenoso. Luego inició con irritabilidad, agresividad, agitación, agnosia nominal, alucinaciones visuales e inversión del ciclo vigilia-sueño (figura 1). Se suspendió la timoglobulina y se continuó con tacrolimus. Los exámenes de laboratorio mostraron linfopenia, sin otros hallazgos anormales (cuadro 1).

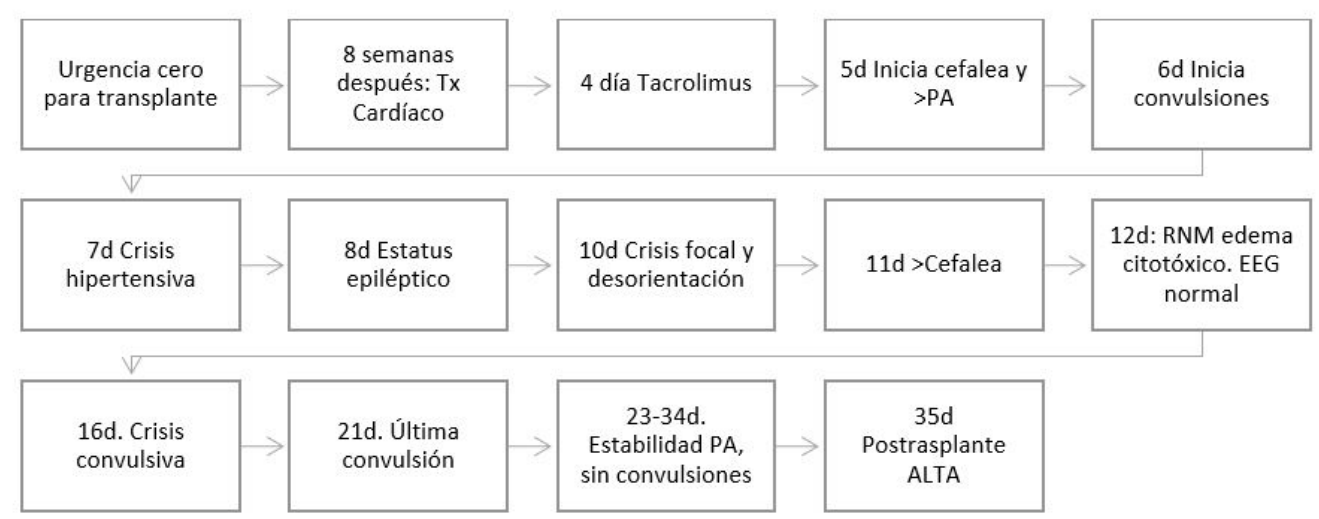

Figura 1. Línea del tiempo a partir de la urgencia cero para trasplante Tx: trasplante; d: día; RNM: resonancia nuclear magnética; EEG: electroencefalograma; >: aumento, PA: presión arterial

Cuadro 1. Resultados de los exámenes de laboratorio al quinto día postrasplante

\begin{tabular}{lc}
\hline Examen & Resultado \\
\hline Hemoglobina & $10,2 \mathrm{~g} / \mathrm{dL}$ \\
\hline Hematocrito & $31,6 \%$ \\
\hline Leucocitos & $5400 \mathrm{por} \mathrm{uL}$ \\
\hline Linfocitos & $778 \mathrm{por} \mathrm{uL}$ \\
\hline Proteína C reactiva-PCR & $3,4 \mathrm{mg} / \mathrm{dL}$ \\
\hline Rangos de glicemia & $77-96 \mathrm{mg} / \mathrm{dL}$ \\
\hline Transaminasa oxaloacética-GOT & $34 \mathrm{U} / \mathrm{L}$ \\
\hline Transaminasa pirúvica-GPT & $59 \mathrm{U} / \mathrm{L}$ \\
\hline Creatinina sérica & $0,28 \mathrm{mg} / \mathrm{dL}$ \\
\hline Nitrógeno ureico-BUN & $6,3 \mathrm{mg} / \mathrm{dL}$ \\
\hline Sodio sérico & $136,2 \mathrm{mEq} / \mathrm{L}$ \\
\hline Potasio sérico & $3,5 \mathrm{mEq} / \mathrm{L}$ \\
\hline Magnesio sérico & $2 \mathrm{mg} / \mathrm{dL}$ \\
\hline Calcio sérico & $1,15 \mathrm{mmol} / \mathrm{L}$ \\
\hline Triglicéridos & $179 \mathrm{mg} / \mathrm{dL}$ \\
\hline Colesterol total & $218 \mathrm{mg} / \mathrm{dL}$ \\
\hline Hormona estimulante de la tiroides-TSH & $2,85 \mathrm{mUl} / \mathrm{mL}$ \\
\hline Tiroxina libre-T4L & $12 \mathrm{ng} / \mathrm{dL}$ \\
\hline Anticuerpos contra citomegalovirus, & $\mathrm{Negativos}$ \\
\hline virus de Epstein-Barr, T. gondii & \\
\hline
\end{tabular}


Se ha sugerido que la neurotoxicidad asociada a los anticalcineurínicos está mediada por una vasculopatía secundaria a la lesión endotelial directa, la cual daña la barrera hematoencefálica, estimula la liberación de sustancias vasoconstrictoras, favorece microangiopatía en la vasculatura cerebral y desencadena edema cerebral vasogénico.
El tacrolimus no fue suspendido por el riesgo de rechazo del injerto con la disminución del inmunosupresor. Con dosis de $3 \mathrm{mg}$ al día de tacrolimus se alcanzaron niveles óptimos de tacrolimus de $8,5 \mathrm{ng} / \mathrm{mL}$ (cuadro 2).

Cuadro 2. Relación entre dosis y niveles sanguíneos de tacrolimus con la biopsia miocárdica y la ecocardiografía según tiempo postrasplante

\begin{tabular}{|c|c|c|c|c|}
\hline $\begin{array}{c}\text { Día } \\
\text { postrasplante }\end{array}$ & $\begin{array}{l}\text { Dosis tacrolimus } \\
\text { oral c/12 horas }\end{array}$ & $\begin{array}{l}\text { Niveles sanguíneos } \\
\text { tacrolimus }(\mathrm{ng} / \mathrm{ml})\end{array}$ & $\begin{array}{l}\text { Biopsia endo } \\
\text { miocárdica }\end{array}$ & $\begin{array}{c}\text { Ecocardiografía } \\
\text { transtorácica }\end{array}$ \\
\hline 6 & $0,5 \mathrm{mg}$ & 2,4 & -- & \\
\hline 8 & 1 & 7,4 & -- & $\begin{array}{l}\text { Disfunción sistólica } \\
\text { leve VD*. TAPSE } 10 . \\
\text { FEVI }^{\diamond} 63 \% \text {. No HTP }\end{array}$ \\
\hline 11 & 1 & 3,5 & -- & -- \\
\hline 12 & 1 & 2,1 & -- & -- \\
\hline 14 & 2 & 6,3 & $\begin{array}{l}\text { Rechazo } \\
\text { celular } \\
\text { grado I }\end{array}$ & \\
\hline 18 & 3 & 8,5 & & $\begin{array}{l}\text { Disfunción sistólica } \\
\text { leve VD*, TAPSE 10, } \\
\text { FEVI } 75 \% \text {. No HTP¥. } \\
\text { Leve hipertrofia bi- } \\
\text { ventricular }\end{array}$ \\
\hline
\end{tabular}

*VD ventrículo derecho †TAPSE: desplazamiento del anillo tricúspideo FEVI fracción de eyección del ventrículo izquierdo łHTP hipertensión pulmonar

Al paciente se le realizaron dos tomografías de cráneo, las cuales fueron normales. Una semana después de haber iniciado el cuadro clínico, una resonancia magnética cerebral mostró edema citotóxico parieto-occipital izquierdo, la cual confirmó el diagnóstico de síndrome de encefalopatía posterior reversible (figura 2). El electroencefalograma de cuatro horas no mostró actividad epileptiforme. Veinte días después del trasplante, el paciente alcanzó cifras tensionales normales, no volvió a presentar delirium o crisis convulsivas, lo que permitió el paso de levetiracetam intravenoso a vía oral, que se suspendió previo al alta por la no recurrencia de episodios ictales, sin evidenciar ningún déficit neurológico, ni alteración afectiva, ni comportamental residual. La medicación al alta fue metoprolol, verapamilo, enalapril, sildenafil, tacrolimus, micofenolato y prednisolona. Tres meses posterior al trasplante se realizó tomografía de craneo de control, la cual fue reportada sin hallazgos patológicos. 
La toxicidad neurológica asociada al tacrolimus pueden tener manifestaciones leves como el temblor distal o graves como estatus epiléptico, alteraciones motoras o del lenguaje. La aparición de los efectos adversos no se relacionan con los niveles en sangre del medicamento, por lo cual se ha propuesto que es un efecto idiosincrático.

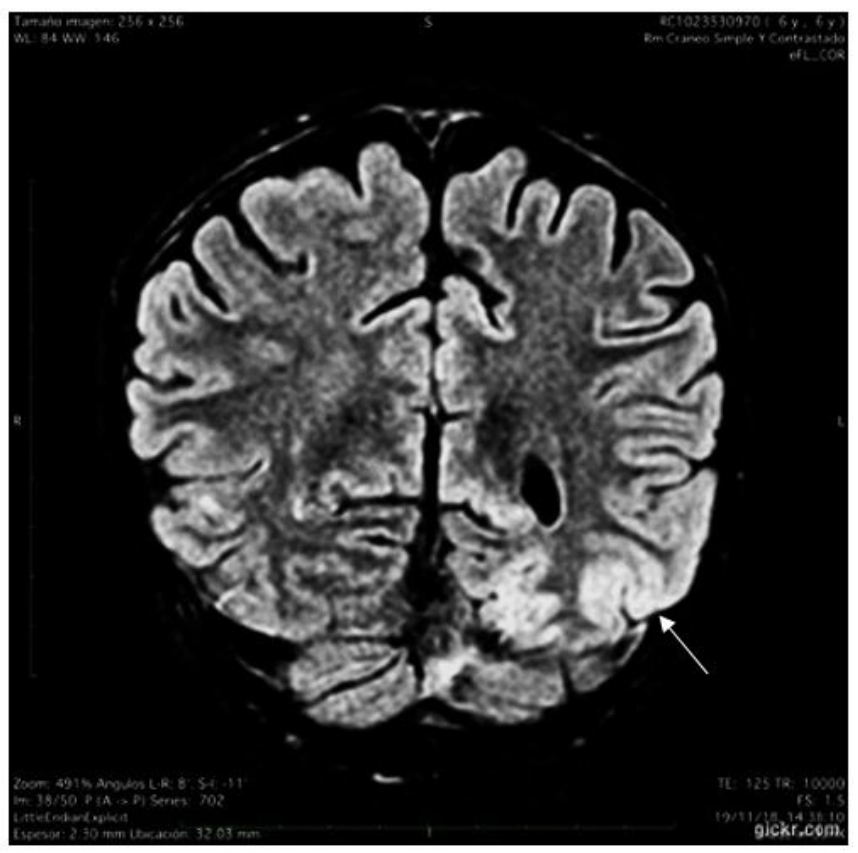

Figura 2. Imagen de resonancia magnética. Secuencia Flair, hiperintensidad parieto-occipital izquierda.

\section{Discusión}

Se presenta un caso de síndrome de encefalopatía posterior reversible en un niño menor de diez años, quien en el postrasplante reciente de corazón, presentó cifras tensionales elevadas, convulsiones y delirium, asociados a hipertensión arterial y uso de tacrolimus.

Los inhibidores de la calcineurina como el tacrolimus y la ciclosporina han transformado positivamente el cuidado de los pacientes trasplantados de corazón al reducir los episodios de rechazo del injerto tanto agudo como crónico $(7,9)$. Dentro de los conocidos efectos adversos del tacrolimus se encuentran la nefrotoxicidad (10), la hipertensión sistémica (11), la neurotoxicidad (12) y la intolerancia a la glucosa (13).

Se ha sugerido que la neurotoxicidad asociada a los anticalcineurínicos está mediada por una vasculopatía secundaria a la lesión endotelial directa que causa el medicamento, la cual daña la barrera hematoencefálica, estimula la liberación de sustancias vasoconstrictoras como la endotelina y el tromboxano, favorece microangiopatía de pequeños vasos en la vasculatura cerebral y finalmente desencadena edema cerebral vasogénico (4). En el paciente presentado, los signos clínicos del síndrome de encefalopatía posterior reversible comenzaron un día después de iniciado el tacrolimus y antes del trasplante no había sufrido de hipertensión arterial, lo cual está a favor del tacrolimus como generador de hipertensión arterial que pudo actuar como un disparador de la disfunción endotelial y del síndrome.

La toxicidad neurológica asociada al tacrolimus pueden tener manifestaciones leves como el temblor distal o graves como estatus epiléptico, alteraciones motoras o del lenguaje (14). La aparición de los efectos adversos no se relacionan con los niveles en sangre del medicamento, por lo cual se ha propuesto que es un efecto idiosincrático (7). En este caso, los niveles séricos del tacrolimus fueron subóptimos y los efectos secundarios de gravedad. 
Dentro de las estrategias recomendadas para el tratamiento del síndrome de encefalopatía posterior reversible se enfatiza la importancia del diagnóstico temprano para prevenir las secuelas como el estatus epiléptico, la isquemia, la hemorragia y la herniación cerebral; además, retirar, disminuir o sustituir el inmunosupresor por otro del mismo grupo farmacológico o disminuir la dosis.
Dentro de las estrategias recomendadas para el tratamiento del síndrome de encefalopatía posterior reversible se enfatiza la importancia del diagnóstico temprano para prevenir las secuelas como el estatus epiléptico, la isquemia, la hemorragia y la herniación cerebral; además, retirar, disminuir o sustituir el inmunosupresor por otro del mismo grupo farmacológico o disminuir la dosis $(1,14)$. En el caso descrito no se realizó ninguna de las opciones anteriores buscando evitar el rechazo agudo del injerto. En cambio, se aumentó la dosis del tacrolimus buscando alcanzar niveles terapéuticos en sangre y se dio manejo a la hipertensión arterial, a los episodios ictales y al delirium. Lo anterior permitió estabilidad en la terapia inmunosupresora, disminución del riesgo de rechazo del injerto y resolución del síndrome.

Se han descrito nueve casos de síndrome de encefalopatía posterior reversible en niños, asociado a trasplante de corazón $(1,3,4)$, los cuales guardan similitud con el reporte actual. Dentro de la búsqueda bibliográfica realizada por los autores, este es el primer caso de estas características reportado en Colombia.

Una de las fortalezas de este reporte de caso es la posibilidad de presentar de forma detallada la línea de tiempo de los eventos clínicos ocurridos en el paciente, lo cual fue posible gracias al estrecho seguimiento clínico que tuvo durante la hospitalización. No se dispone de una resonancia cerebral de control que permita comparación con los hallazgos iniciales. El niño actualmente se encuentra asintomático desde el punto de vista cardiovascular y neurológico.

Para el reporte del caso se tuvo en cuenta las pautas CIOMS 2016 (15). Se tomó consentimiento informado a la madre y se pidió autorización al Comité de Ética Institucional.

\section{Conclusión}

El síndrome de encefalopatía posterior reversible debe ser considerado en pacientes trasplantados de corazón que presentan alteraciones neuropsiquiátricas.

\section{Bibliografía}

1. Eilers B, Albers E, Law Y, McMullan DM, Shaw D, Kemna M. Posterior reversible encephalopathy syndrome after pediatric heart transplantation: Increased risk for children with preexisting Glenn/Fontan physiology. Pediatr Transplant. 2016;20(4):552-8.

2. Hinchey J, Chaves C, Appignani B, Breen J, Pao L, Wang A, et al. A Reversible Posterior leukoencephalopathy syndrome. N Engl J Med.1996:334(8):494-500.

3. Araz C, Camkiran A, Zeyneloglu P, Sezgin A, Moray G, Pirat A, et al. Early-onset posterior reversible encephalopathy syndrome after solid organ transplantation in pediatric patients: a report of 2 cases. Transplant Proc. 2013;45(10):3555-7.

4. Loar RW, Patterson MC, O'Leary PW, Driscoll DJ, Johnson JN. Posterior reversible encephalopathy syndrome and hemorrhage associated with tacrolimus in a pediatric heart transplantation recipient. Pediatr Transplant. 2013;17(2):E67-70.

5. Servillo G, Bifulco F, De Robertis E, Piazza O, Striano P, Tortora F, et al. Posterior reversible encephalopathy syndrome in intensive care medicine. Intensive Care Med. 2007;33(2):230-6. 
6. Mak A, Chan BPL, Yeh IB, Ho RCM, Boey ML, Feng PH, et al. Neuropsychiatric lupus and reversible posterior leucoencephalopathy syndrome: a challenging clinical dilemma. Rheumatol Oxf Engl. 2008;47(3):256-62.

7. Kapoor A, Birks E, Lenneman A, McCants K. Posterior Reversible encephalopathy syndrome after heart transplantation: diagnosis and immunosuppressive therapy. Tex Heart Inst J. 2017;44(3):205-8.

8. Gagnier JJ, Kienle G, Altman DG, Moher D, Sox H, Riley D, et al. The CARE Guidelines: Consensus-based clinical case reporting guideline development. Glob Adv Health Med. 2013;2(5):38-43.

9. Colombo D, Ammirati E. Cyclosporine in transplantation - a history of converging timelines. J Biol Regul Homeost Agents. 2011;25(4):493-504.

10. Randhawa PS, Starzl TE, Demetris AJ. Tacrolimus (FK506)-Associated Renal Pathology. Adv Anat Pathol.1997;4(4):265-76.

11. Hoorn EJ, Walsh SB, McCormick JA, Fürstenberg A, Yang C-L, Roeschel T, et al. The calcineurin inhibitor tacrolimus activates the renal sodium chloride cotransporter to cause hypertension. Nat Med. 2011;17(10):1304-9.

12. Bechstein WO. Neurotoxicity of calcineurin inhibitors: impact and clinical management. Transpl Int Off J Eur Soc Organ Transplant. 2000;13(5):313-26.

13. First MR, Gerber DA, Hariharan S, Kaufman DB, Shapiro R. Posttransplant diabetes mellitus in kidney allograft recipients: incidence, risk factors, and management. Transplantation. 2002;73(3):379-86.

14. Ramirez R, Muskula PR, Everley MP. Posterior reversible encephalopathy syndrome after orthotopic heart transplantation: a case report. Am J Case Rep. 2017;18:487-90.

15. World Health Organization, Council for International Organizations of Medical Sciences. International ethical guidelines for health-related research involving humans. Geneva: CIOMS; 2017. 